\title{
The Effect of Motor Imagery on Spinal Segmental Excitability
}

\author{
Sheng Li, ${ }^{1}$ Derek G. Kamper, ${ }^{2,3}$ Jennifer A. Stevens, ${ }^{4}$ and William Z. Rymer ${ }^{2,3}$ \\ ${ }^{1}$ Department of Physical Therapy, University of Montana, Missoula, Montana 59812, ${ }^{2}$ Department of Physical Medicine and Rehabilitation, Feinberg School \\ of Medicine, Northwestern University, Chicago, Illinois 60611-3008, 33ensory Motor Performance Program, Rehabilitation Institute of Chicago, Chicago, \\ Illinois 60611-2654, and ${ }^{4}$ Department of Psychology, College of William and Mary, Williamsburg, Virginia 23187-8795
}

The purpose of this study was to investigate the effect of motor imagery on spinal segmental excitability by recording the reflex responses to externally applied stretch of the extrinsic finger flexors and extensors during the performance of an imaginary task. Nine young healthy subjects performed a series of imagined flexion-extension movements of the fingers. Muscle stretch was imposed concurrently by applying rotations of the metacarpophalangeal joints at 100,300 , or $500 \%$ sec. Three of the nine tested subjects also generated 0.2 Newton meter voluntary flexion torque in preloading tasks before stretch.

At $300 \% \mathrm{sec}$ stretch, electromyogram (EMG) and torque reflex responses, which were observed in the finger flexors in four of nine subjects during motor imagery, were activated at a short latency $(38.6 \pm 10.6 \mathrm{msec})$. This latency was similar to that recorded during a stretch of preactivated flexor muscles $(34.4 \pm 3.6 \mathrm{msec})$, in which motoneurons are already suprathreshold and in which monosynaptic effects of muscle afferents are likely to be discernable. In a similar manner, for stretches imposed at $500 \% \mathrm{sec}$, responses to stretch of the flexors were observed in all five tested subjects in imaginary flexion tasks at very short latencies $(26.4 \pm 3.7 \mathrm{msec})$, again similar to those induced by tendon taps $(22.8 \pm 1.2 \mathrm{msec})$. No EMG response was observed at rest during stretches.

These observations support the view that effects must have been mediated by imagery-related subthreshold activation of spinal motoneurons and/or interneurons, rather than by long-latency transcortical reflex responses. We conclude that motor imagery has a potent effect on the excitability of spinal reflex pathways.

Key words: motor imagery; stretch reflex; spinal excitability; motor; fingers; human

\section{Introduction}

A general feature of motor imagery is that subjects feel they are performing a certain movement without executing it (Jeannerod, 1995; Decety, 1996a; Facchini et al., 2002). Extensive evidence in the literature shows that motor imagery shares a number of neural substrates with those neural centers involved in the actual movement (Decety, 1996a; Crammond, 1997; Abbruzzese et al., 1999). Imaginary movements share additional features with actual movements such as kinematic constraints (Sirigu et al., 1995), temporal properties (Decety et al., 1989; Sirigu et al., 1995), and the effects on motor performance (Yue and Cole, 1992). Furthermore, transcranial magnetic stimulation (TMS) studies have demonstrated an increased excitability in primary motor cortex (M1) and corticospinal pathways during motor imagery when compared with the resting state, as evidenced by a decrease in motor threshold and an increase in size of the motor evoked potentials (MEPs) (Kasai et al., 1997; Kiers et al., 1997; Fadiga et al., 1999; Yahagi and Kasai, 1999; Facchini et al., 2002, Li et al., 2004). However, the MEPs do not provide information about the level of the corticospinal pathway at which these effects arise (Petersen et al., 2003). Contributions to the in-

Received July 12, 2004; revised Sept. 12, 2004; accepted Sept. 13, 2004.

This work was supported by National Institute on Disability and Rehabilitation Research Training Grant H133P990006 (S.L.)

Correspondence should be addressed to Dr. Sheng Li, Department of Physical Therapy, University of Montana, Skaggs Building, Room 135, Missoula, MT 59812. E-mail: sheng.li@umontana.edu. DOI:10.1523/JNEUROSCI.2781-04.2004

Copyright $\odot 2004$ Society for Neuroscience $\quad$ 0270-6474/04/249674-07\$15.00/0 creased amplitude of the MEPs may arise from the spinal motoneurons (Morita et al., 2000) or from spinal interneurons (INs), which receive direct inputs from $\mathrm{M} 1$ or linked premotor areas (Dum and Strick, 1991; He et al., 1995).

Controversy exists in the literature regarding the relative contributions of supraspinal and spinal changes in excitability to the observed increase in the MEP amplitudes. Both H-reflex and F-wave techniques, in combination with TMS stimulation over M1, have been used to explore these contributions. Some researchers have found no effect of motor imagery on H-reflex amplitude [extensor carpi radialis (Kasai et al., 1997; Hashimoto and Rothwell, 1999) and flexor digitorum superficialis (Abbruzzese et al., 1996)], whereas others have found a facilitatory effect (flexor carpi radialis) in half of their subjects, resulting in a statistically significant difference (Kiers et al., 1997). Rossini et al. (1999) reported specific facilitation of the F waves recorded from intrinsic hand muscles (abductor digiti minimi, first dorsal interosseous) during motor imagery. In the soleus muscles, Bonnet et al. (1997) reported a similar increase in excitability (based on H-reflexes and tendon reflexes) during motor imagery. Facilitation of these reflexes suggests subthreshold excitation of the spinal motoneuron pool of the target muscle during motor imagery.

In contrast, there was no change in the F waves in another motor imagery study, also recorded from intrinsic hand muscles (Facchini et al., 2002). One limitation of the F-wave technique is that modulation of $\mathrm{F}$ waves may not be representative of the change of the entire $\alpha$ motoneuron pool (Mercuri et al., 1996). Thus, the effect of motor imagery at the spinal level remains unclear. 
Stretch reflexes rely on both monosynaptic and polysynaptic connections within the spinal cord and may also involve a transcortical loop (Abbruzzese et al., 1985; Goodin et al., 1990; Capaday et al., 1991), reflecting the excitability of the spinal interneurons as well as motoneurons. Examination of the effects of motor imagery on the stretch reflex response may help determine whether motor imagery has discernable effects on spinal excitability.

In the present study, we hypothesized that motor imagery effects on modulation of corticospinal excitability could also be recorded at the segmental level. We examined this effect by comparing reflex responses [electromyogram (EMG) or joint torque] to externally imposed stretches of finger flexors and extensors at rest with those recorded during motor imagery. We found that the earliest effects of motor imagery were usually discernable at relatively short latencies (average, $26.4 \pm 3.7 \mathrm{msec}$ ), a time too short to allow significant participation of long-loop pathways. Furthermore, examination of the trial-to-trial variation in response showed patterns of EMG response that were more likely to reflect changes in baseline motoneuronal and/or interneuronal excitability than changes in long-loop pathways.

\section{Materials and Methods}

Subjects. Nine healthy volunteers, all males, participated in the experiments. All of them were right-handed according to their preferential use of the right-hand during writing and eating. The mean age of the subjects was $30 \pm 4.9$ (mean $\pm S D$ ) years. Their weight was $67 \pm 10.5 \mathrm{~kg}$, and their height was $1.72 \pm 0.04 \mathrm{~m}$. The left arm of each subject was used for testing. All subjects gave informed consent before the experiment according to the procedures approved by the Institutional Review Board at Northwestern University.

Apparatus and recordings. Stretches were provided by a servomotor (1.4 horsepower; PMI Motion Technologies, Radford, VA) as described previously (Kamper and Rymer, 2000). Briefly, the subject was positioned on an adjustable chair such that the shoulder was abducted $\sim 50^{\circ}$ and the elbow was flexed $\sim 90^{\circ}$. The forearm and wrist were placed in a fiberglass cast and secured in the neutral position to a clamp attached to the table to which the motor was secured. This arrangement prevented translation and rotation of the arm. The motor shaft extended vertically above the table. The metacarpophalangeal (MCP) joints were aligned with the shaft such that rotation of the shaft produced an equivalent angular rotation of the MCP joints. An aluminum channel was connected to the shaft and to a $\mathrm{U}$-shaped piece into which the fingers were placed. A bead-filled bladder surrounding the fingers was attached to the inner surface of the U-shaped piece. Evacuation of the bladder with a vacuum pump provided a rigid coupling between the fingers and the U-shaped piece and, thus, the motor shaft. A strap kept the thumb extended and abducted from the palm so it did not interfere with finger flexion.

Differential surface EMG electrodes (DelSys, Boston, MA) were mounted above the muscle bellies of the flexor digitorum superficialis (FDS) and the extensor digitorum communis (EDC) of the left arm to record muscle activity. The EMG signals were amplified and low-pass filtered at $225 \mathrm{~Hz}$. The signals from the left forearm (both FDS and EDC) were displayed on-line on an oscilloscope $\sim 1.5 \mathrm{~m}$ in front of the subject. EMG signals were set at a high gain $(25 \mu \mathrm{V}$ per division $)$ to ensure complete EMG silence during motor imagery.

Angular position, rotational velocity, and torque were measured throughout the trials with a position encoder (138647; PMI Motion Technologies), tachometer (PMI Motion Technologies), and torque transducer (TRT-200; Transducer Techniques, Temecula, CA), respectively. All signals were sampled at $500 \mathrm{~Hz}$ using customized software, and the data were saved for off-line analysis.

Procedures. Two experimental conditions were investigated in all nine subjects: rest (the absence of imagery) and motor imagery. As shown in Figure 1, there were four different motor imagery tasks: ImFlex-EXT, ImExt-EXT, ImFlex-FLEX, and ImExt-FLEX, where ImFlex/Ext stands

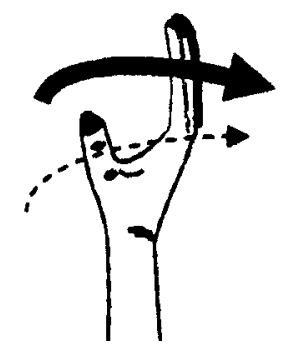

ImExt-EXT

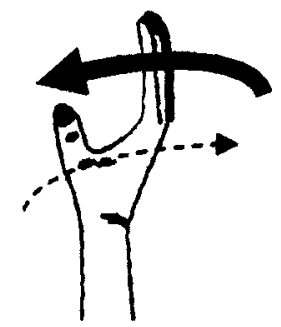

ImExt-FLEX

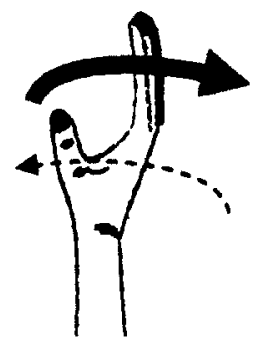

ImFlex-EXT

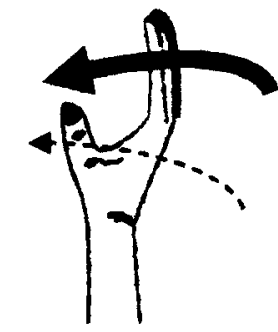

ImFlex-FLEX

Figure 1. Illustration of four motor imagery tasks. FLEX/EXT, Flexion/extension stretches between $30^{\circ}$ of flexion and $0^{\circ}$ of the MCP at either 300 or $100 \%$ sec. Solid arrow, Direction of stretch; dashed arrow, direction of imagined movement. Note that the hand-finger configuration is not the same as that tested in the experiment.

for the direction of imagery and EXT/FLEX for the direction of stretches imposed by the MCP rotator. Subjects were instructed to keep their muscles relaxed before the stretch. Stretches were performed at 300 or $100 \% \mathrm{sec}$, with a rise time equal to $10 \%$ of the total movement time. The range of stretch was from 0 to $30^{\circ}$ of flexion of the MCP joints for the FLEX stretch, whereas it was from $30^{\circ}$ of flexion to $0^{\circ}$ for the EXT stretch. The ranges were selected such that the flexors and extensors were equally stretched with respect to the neutral position of MCP joints. There are no existing data on the respective reflex thresholds of FDS and EDC, but because both muscles are near optimal fiber length for these postures (Kamper et al., 2001), we expected that the effects on the muscles would be similar. In addition, two tasks were also tested in five of nine subjects, ImFlex-EXT and at rest using fast stretches $\left(500^{\circ} / \mathrm{sec}\right)$, at which the MCP joints were rotated from $20^{\circ}$ of flexion to $0^{\circ}$, resulting in a movement time of $40 \mathrm{msec}$.

A "ramp-hold- ramp" stretch was used (Fig. 2). In tasks at rest, the EXT stretch was imposed during the first half of the trial, whereas the FLEX stretch was applied during the second half of the trial.

The instruction for motor imagery was to mentally extend-flex the secured left fingers against the U-shaped piece as hard as possible and to maintain the imagined contraction until the end of the actual stretch. The stretch was initiated unexpectedly within $3-5 \mathrm{sec}$ after the verbal command to begin the imagery. Subjects were asked to practice the mental tasks for a few minutes before testing to ensure that they were able to keep the EMG silent during motor imagery. EMG silence was defined as the absence of any background activity at the sensitivity of $25 \mu \mathrm{V}$ per division. During motor imagery, the subject was also instructed to keep the whole body still, including the other hand.

The order of conditions was randomized. Five trials were recorded for each task. The interval between two consecutive trials was $\sim 20 \mathrm{sec}$. The experimenter discarded a motor imagery trial if EMG activities were detected visually during the experiment and a repeat trial was performed.

Three of nine tested subjects were randomly selected to perform additional preloading tasks in an effort to gauge the magnitude of the imagery effect. In the preloading tasks, subjects generated 0.2 Newton meter $(\mathrm{Nm})$ of flexion torque against the motor $(\sim 1.5 \% \mathrm{MVC})$. Under the 


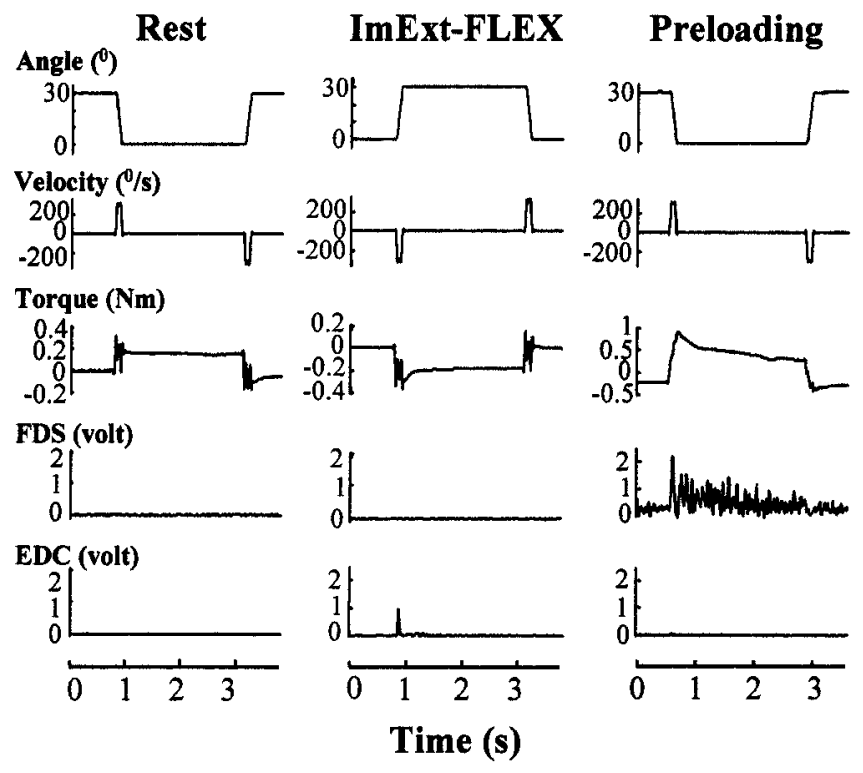

Figure 2. Typical traces from a representative subject during different tasks at $300 \% \mathrm{sec}$ stretches: rest (left), ImExt-FLEX (middle), and preloading (right). No torque and EMG responses were observed at rest. EMG responses in the EDC were evident during ImExt-FLEX tasks. EMG and torque reflex responses were present during preloading tasks. Note that positive position and negative torque denote flexion; EMG signals were rectified, filtered signals.

preloading condition, the subject received a "do not intervene voluntarily" instruction (Crago et al., 1976) (i.e., the subject attempted to maintain the same level of voluntary activation as required to attain $0.2 \mathrm{Nm}$ of flexion torque before the stretch).

To estimate the latency of monosynaptic pathways to motoneurons innervating finger flexors, tendon tap tests were performed in three randomly selected subjects. The left forearm was supported on the table in a supinated position with the elbow placed at $\sim 90^{\circ}$ of flexion. The subjects were asked to relax with their fingers naturally curved. A sharp tap on the FDS tendons, medial to the tendon of palmaris longus at $\sim 1 \mathrm{~cm}$ proximal to the wrist joint, was applied using a customized tapper equipped with a Kistler (Don Mills, Ontario, Canada) force sensor. The tapper allowed measurement of the onset and impact force of the tendon tap.

Data analysis. Two main parameters, magnitude of torque-EMG responses and latency of reflex EMG responses, were calculated. Magnitudes of torque and EMG responses were calculated to evaluate the global effects of motor imagery, whereas the onset latencies of stretch reflexes were used to examine origins of the effects (i.e., transcortical or spinal).

Magnitude of torque/EMG response. Torque, angle, and angular velocity data were digitally low-pass filtered at $25 \mathrm{~Hz}$ using a second-order, zero-lag Butterworth filter. The EMG signals were rectified, and the resulting signals were low-pass filtered at $25 \mathrm{~Hz}$ to create envelopes used to quantify muscle activity.

First, the onset $\left(t_{0}\right)$ and the end $\left(t_{1}\right)$ of stretch were determined by the time when the velocity reached or returned to $10 \%$ of its maximum value $(500,300$, or $100 \% \mathrm{sec})$. The background torque $\left(\mathrm{T}_{\mathrm{BG}}\right)$ and the background $\mathrm{EMG}\left(\mathrm{EMG}_{\mathrm{BG}}\right)$ were defined as the mean values calculated from $t_{0}-100 \mathrm{msec}$ to $t_{0}$. The torque responses were defined as the difference between the peak torque during the stretch and $\mathrm{T}_{\mathrm{BG}}$. The peak torque was calculated as the torque averaged over a $25 \mathrm{msec}$ window, centered about the instance of the maximal torque during the stretch period (from $t_{0}$ to $t_{1}$ ). For tasks at rest, the torque responses were calculated for the two halves separately (Fig. 2) and used as a baseline for imagery tasks with EXT (ImFlex-EXT, ImExt-EXT) and FLEX (ImFlex-FLEX, ImExtFLEX) stretches, respectively. Similarly, the peak EMG magnitude $\left(E G_{P K}\right)$ was obtained from the rectified, low-pass filtered EMG signals during the stretch period. The magnitude of EMG response was defined as the difference between the peak EMG value and $\mathrm{EMG}_{\mathrm{BG}}\left(\mathrm{EMG}_{\mathrm{PK}}-\mathrm{EMG}_{\mathrm{BG}}\right)$.

The onset latency of stretch reflex. To avoid the potential prolongation of the latency by signal processing, only rectified, but unfiltered, signals of raw EMG data were used. A reflex EMG response was considered to have occurred when the peak EMG magnitude $\left(\mathrm{EMG}_{\mathrm{PK}}\right)$ during the stretch exceeded three SDs of the $\mathrm{EMG}_{\mathrm{BG}}$. The latency of the stretch reflex was computed as the time interval from the movement onset $\left(t_{0}\right)$ to the moment when the EMG magnitude exceeded two SDs of the baseline. EMG responses were analyzed for extensors (EDC) and flexors (FDS) separately. Similarly, the latency for the tendon tap tests was calculated as the interval between the onset of the tap $\left(t_{0}\right)$ and the EMG onset. The EMG response was also visually inspected trial by trial.

Statistics. The data here are presented as means, whereas the figures show means and SE bars. Repeated measures ANOVAs were used with factors IMAG (three levels: None (rest), ImFlex, and ImExt) and STRE (two levels: flexion and extension). Whenever necessary, post hoc Tukey's honest significant difference tests were used to compare the various levels of a factor. Paired Student's $t$ tests were used to test resting conditions.

\section{Results}

In the present study, we focused on motor imagery tasks that were accompanied by finger stretches applied at several different velocities. At $300^{\circ} / \mathrm{sec}$, the duration of the imposed movement was $\sim 100 \mathrm{msec}$, a time span less than the minimum required for a voluntary reaction time movement, thereby allowing us to focus on reflex rather than voluntary reaction-time responses. Trials at $100 \% \mathrm{sec}$ were also analyzed and revealed a similar pattern of torque and EMG responses. Background EMG and torque values were analyzed and showed no difference across different conditions of imagery tasks and rest within the same subjects, indicating that comparisons of EMG responses across trials were legitimate. High-speed stretches $\left(500^{\circ} / \mathrm{sec}\right)$ were used to investigate the effect of stretch velocity on reflex latency during motor imagery.

\section{Effects of motor imagery on reflex EMG responses in forearm muscles}

\section{Pattern of reflex EMG responses}

In general, reflex EMG responses were recorded during most imagery tasks, whereas no response was recorded at rest in any of the subjects across different stretch velocities (Fig. 2). Furthermore, the EMG responses showed a novel pattern during motor imagery tasks in that the responses were much more evident during the FLEX stretches than during the EXT stretches.

As summarized in Table 1, reflex EMG responses (peak EMG magnitude more than three SDs of background EMG) from EDC occurred in all nine subjects during the ImExt-FLEX tasks and in seven of nine subjects during the ImFlex-FLEX tasks. In these tasks (FLEX stretches), reflex EMG responses were present when EDC was stretched, regardless of the direction of imagined movements. In contrast, reflex EMG responses from FDS were observed in four of nine subjects during the ImFlex-EXT tasks in which this muscle was the putative primary mover in the imaginary movement and was stretched (EXT) by the actuator. No reflex responses were observed in the ImExt-EXT tasks where FDS was stretched when EDC was the putative primary mover of the imagined movement.

\section{Magnitude of EMG response}

Magnitudes of responses were quantified by repeated-measure ANOVAs. A $2 \times 3$ two-way ANOVA (STRE $\times$ IMAG) was performed to compare the magnitude of EMG responses $\left(\mathrm{EMG}_{\mathrm{PK}}-\right.$ $\mathrm{EMG}_{\mathrm{BG}}$ ) in EDC within the same subject across different conditions (motor imagery, rest). The results showed a significant effect of IMAG $\left(F_{(2,16)}=4.13 ; p<0.05\right)$ and a significant interaction of IMAG $\times \operatorname{STRE}\left(F_{(2,16}=4.14 ; p<0.05\right)$, indicating that 
Table 1. Patterns of EMG response to $300 \% / \mathrm{s}$ stretches

\begin{tabular}{|c|c|c|c|c|c|c|c|c|c|c|}
\hline \multirow[b]{2}{*}{ No. } & \multicolumn{2}{|l|}{ Rest } & \multicolumn{2}{|c|}{ ImFlex-EXT } & \multicolumn{2}{|c|}{ ImExt-EXT } & \multicolumn{2}{|c|}{ ImFlex-FLEX } & \multicolumn{2}{|c|}{ ImExt-FLEX } \\
\hline & FDS & $\mathrm{EDC}$ & FDS & EDC & FDS & $\mathrm{EDC}$ & FDS & $\mathrm{EDC}$ & FDS & EDC \\
\hline 1 & - & - & $22-26$ & - & - & - & - & - & - & $50-78$ \\
\hline 2 & - & - & - & - & - & - & - & $62-79$ & - & $63-82$ \\
\hline 3 & - & - & - & - & - & - & - & $54-72$ & - & $69-83$ \\
\hline 4 & - & - & - & - & - & - & - & $46-67$ & - & $60-79$ \\
\hline 5 & - & - & - & - & - & - & - & $35-47$ & - & $51-63$ \\
\hline 6 & - & - & $34-61$ & - & - & - & - & $65-72$ & - & $69-77$ \\
\hline 7 & - & - & $31-72$ & - & - & - & - & - & - & $54-72$ \\
\hline 8 & - & - & $31-40$ & - & - & - & - & $61-77$ & - & $53-63$ \\
\hline 9 & - & - & - & - & - & - & - & $48-56$ & - & $43-57$ \\
\hline
\end{tabular}

The reflex latency (in milliseconds) varies across trials within a subject. No., Subject number; ImFlex/Ext, imagined flexion/extension movements; FLEX/EXT, flexion/extension stretches; FDS, flexor digitorum superficialis; EDC, extensor digitorum communis; numbers range of the latency (in milliseconds); - , no reflex response. Note that the criterion for reflex response is peak EMG magnitude $>3$ SDs of background EMG.

EMG responses from EDC were significantly larger during motor imagery in the flexion stretch (ImFlex-FLEX, 0.29 V; ImExtFLEX, $0.25 \mathrm{~V}$ ) than in other conditions (ImFlex-EXT, ImExtEXT, Rest, $0.01 \mathrm{~V}$ ). The same type of ANOVA was performed for EMG responses from FDS and only showed a significant effect of $\operatorname{IMAG}\left(F_{(2,16)}=3.85 ; p<0.05\right)$. Tukey's post hoc test revealed that the EMG response in FDS was significantly larger in ImFlexEXT tasks $(0.15 \mathrm{~V})$ than in other tasks (ImExt-EXT, $0.04 \mathrm{~V}$; ImExt-FLEX, ImFlex-FLEX, 0.01 V; Rest, 0.01 V).

When normalized by the EMG response obtained from the voluntary preloaded condition in which the joint was stretched at $300^{\circ} / \mathrm{sec}$, the averaged EMG magnitude in three subjects was $48.7 \%$ for the ImFlex-EXT tasks. The averaged magnitude of EMG response was $89.5 \%$ for the preloading tasks stretched at $100^{\circ} / \mathrm{sec}$.

\section{The onset latency of stretch reflex}

Latency of EMG onset for stretch reflex was dependent on imagined movement and muscle (Table 1); it was shorter in FDS than in EDC. Furthermore, the reflex responses were seen in both FDS and EDC during imagined flexion, whereas only responses in EDC were observed for imagined extension. For stretches imposed at $300 \% \mathrm{sec}$, the latency of onset for stretch responses ranged from 22 to $72 \mathrm{msec}$ in the finger flexors and from 35 to 83 msec in the finger extensors (Table 1). On average, the EMG latency was $38.6 \mathrm{msec}(\mathrm{SD}, \pm 10.6)$ in the FDS for the ImFlex-EXT tasks, $58.1 \mathrm{msec}( \pm 11.5)$ in the EDC for the ImFlex-FLEX tasks, and $65.4 \mathrm{msec}( \pm 9.8)$ in the EDC for the ImExt-FLEX tasks. The averaged latency was $34.4 \mathrm{msec}( \pm 3.6)$ in the FDS during the preloading tasks, ranging from 25.8 to $50 \mathrm{msec}$. As shown in Table 1, the latency varied across trials within a subject. The shortest latencies for the ImFlex-EXT tasks in each subject (22 to $34 \mathrm{msec}$ ) (Table 1) were comparable with those observed during preloading tasks in which the same muscles were stretched $(34.4 \pm 3.6 \mathrm{msec})$. If only the shortest latency was considered for each subject, the average was $29.5 \mathrm{msec}$.

To assess the effects of stretch velocity on the onset latency, the latencies were compared at different movement velocities. Unlike the responses for $300 \% \mathrm{sec}$ stretch, EMG responses in FDS were observed at stretch velocities of $500 \%$ sec in all five tested subjects in the ImFlex-EXT task, whereas no EMG response was observed at rest. Interestingly, in one of the five subjects, EMG response in FDS was elicited during fast stretch $(500 \% \mathrm{sec})$ but was not observed in the same ImFlex-EXT task during the stretch at $300 \%$ sec.

Furthermore, the latency decreased substantially below that at $300^{\circ} / \mathrm{sec}$, ranging from 21 to $40 \mathrm{msec}$ with an average of $26.4 \mathrm{msec}$ $( \pm 3.7)$. In addition, the onset latency within a subject was less

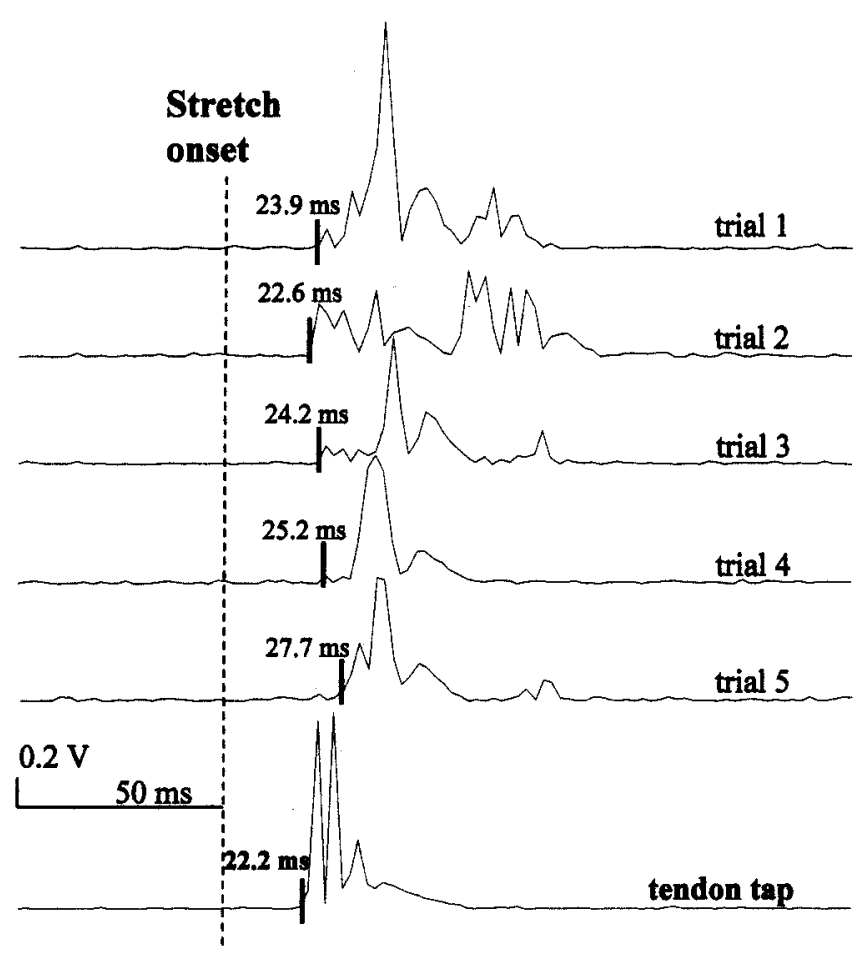

Figure 3. Individual trials of ImFlex-EXT tasks at $500 \% \mathrm{sec}$ and a tendon tap trial from a representative subject. Trials are aligned with the stretch (tap) onset. Note that the latency at $500 \%$ sec is comparable with that for the tendon tap reflex. Traces, Rectified, unfiltered EMG signals; dotted vertical line, stretch onset; solid vertical line, onset of EMG response.

variable under the $500^{\circ} / \mathrm{sec}$ stretch (Fig. 3). Of particular interest, this latency was very close to that measured from tendon tap reflex tests (average, $22.8 \pm 1.2 \mathrm{msec}$; range, $20-24 \mathrm{msec}$ ).

\section{Effects of motor imagery on reflex torque responses}

Predictably, the presence of EMG responses was accompanied by changes in torque responses. As shown in the top panel of Figure 4 , the torque response averaged across subjects during the stretch period was larger in the ImFlex-EXT tasks $(0.26 \mathrm{Nm})$ than in the ImExt-EXT tasks $(0.2 \mathrm{Nm})$ and at rest $(0.2 \mathrm{Nm})\left(F_{(2,16)}=6.21\right.$; $p<0.01)$. Note that if the subjects without reflex EMG responses were excluded for the ImFlex-EXT tasks, the averaged peak was even larger $(0.36 \mathrm{Nm})$.

In contrast, no difference in torque responses was observed in motor imagery tasks with FLEX stretches between ImExt-FLEX $(0.25 \mathrm{Nm})$ and ImFlex-FLEX $(0.23 \mathrm{Nm})$ tasks. However, the 


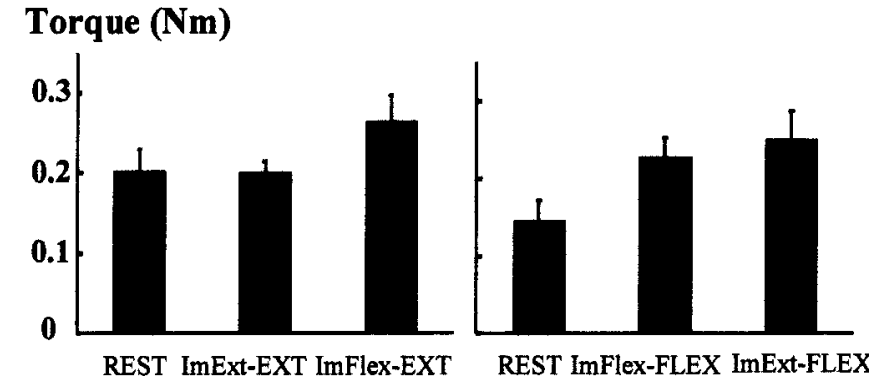

Figure 4. Torque responses to external stretches at rest and during motor imagery tasks are presented with SEs. When the flexors were stretched (left), torque responses in the ImFlex-EXT tasks were significantly larger than those in the ImExt-EXT and at rest. As shown to the right, torque responses to the stretch of the extensors were significantly larger in motor imagery tasks than at rest. No difference was found between ImFlex-FLEX and ImExt-FLEX tasks.

torque responses during imagery tasks with FLEX stretches were both significantly larger than those at rest $(0.15 \mathrm{Nm})$ during the second half of the tasks $\left(F_{(2,16)}=7.04 ; p<0.006\right)$ (Fig. 4, bottom panel). Based on Student's $t$ tests, the torque response at rest was larger in the first half $(0.2 \mathrm{Nm})$ than in the second half $(0.15 \mathrm{Nm})$ of the trial $(p<0.001)$.

The torque responses observed during motor imagery tasks were small in comparison to those that could be seen during the preloading conditions in three tested subjects. Averaged peak torque responses in three subjects were $0.73 \mathrm{Nm}$ for trials stretched at $100 \% \mathrm{sec}$ and $0.92 \mathrm{Nm}$ at $300^{\circ} / \mathrm{sec}$.

\section{Discussion}

\section{Effects of motor imagery on the stretch reflex}

We focus discussion on the imagery-related modulation of stretch reflex in the finger flexors, because reflex responses in the flexors were compared during motor imagery, muscle activation, and tendon taps. The results obtained from this study show clearcut modulation of stretch reflex excitability during motor imagery. EMG responses and accompanying torque responses to the muscle stretch during motor imagery were greatly enhanced when compared with the responses collected without imagery (Figs. 2-4). Additionally, the latency of the response was quite short and similar to that observed during $300 \% \mathrm{sec}$ stretch of voluntarily preactivated muscle. This indicates that there was unlikely to be sufficient time for voluntary reaction time responses to influence the EMG response. More importantly, the shortest latencies for the imaginary tasks observed during fast stretches at $500 \% \mathrm{sec}$ was similar to those induced by tendon taps, thus providing compelling evidence that imagery-related modulation of stretch reflex occurs at the spinal level. Furthermore, close similarity in reflex responses recorded during tasks performed using preloading, and during motor imagery tasks supports the notion that motor imagery shares many features with actual movements (Decety, 1996a; Crammond, 1997; Abbruzzese et al., 1999).

There is general agreement that the motor cortex increases its excitability to magnetic stimulation during motor imagery (Kasai et al., 1997; Kiers et al., 1997; Fadiga et al., 1999; Yahagi and Kasai, 1999; Facchini et al., 2002). It is likely then that modulation of imagery-induced reflex response originates at a supraspinal level where the motor outflow arises.

However, our observations strongly favor the hypothesis that spinal excitability is increased broadly over the period of imaginary movement and not linked closely to enhanced cortical excitability via a long-loop mechanism. As summarized in Table 1, the EMG latency varied considerably across trials within a sub- ject, in which reflexes were examined using $300 \%$ sec stretches. Furthermore, the shortest response latencies in FDS in the ImFlex-EXT tasks were comparable with those observed in the preloading tasks at the same stretches $\left(300^{\circ} / \mathrm{sec}\right)$ to the same muscle (FDS). During stretch of preactivated muscle, motoneurons are already suprathreshold, and monosynaptic effects of muscle afferents are likely to be discernable. Therefore, the results of within-subject variation at $300 \% \mathrm{sec}$ stretch could best be explained by hypothesizing that motoneuron and/or interneuron pools of the target muscle are excited to different and potentially time-varying subthreshold levels during motor imagery. Consequently, stretch-induced afferent inputs elicit reflex responses at different latencies in different trials. In contrast, within-subject variations could also be partly attributed to possibility of nonhomogeneous mental performance of the subjects because of the lack of external cueing (e.g., modulated pitch) (cf. Fadiga et al., 1995).

More importantly, the onset latency discerned during $500 \%$ sec provides convincing evidence that the imagery-related modulation of stretch reflex responses most likely occurs at the spinal level. During fast stretches $(500 \% \mathrm{sec})$, the earliest discernable onset latencies in FDS (average, $26.4 \mathrm{msec}$, from 21 to $40 \mathrm{msec}$ ) were close to those induced by tendon taps $(22.8 \mathrm{msec}$, from 20 to 24 $\mathrm{msec})$. The difference is presumably attributable to the fact that the angular joint perturbation was not as abrupt as that achieved during tendon tap and to the fact that excitability may vary throughout the imaginary task. Furthermore, short onset latencies $(26.4 \mathrm{msec})$ of EMG responses during fast stretches in the imagery tasks were similar to those for short latency reflexes or so called M1 in other hand and forearm muscles [flexor pollicus longus, $24 \pm 2 \mathrm{msec}$ (Marsden et al., 1976); flexor carpi radialis, 17-26 msec (Lewis et al., 2004); 20-40 msec (Lin and Sabbahi, 1998)]. Thus, such latencies appear too short for potential involvement of cortical long-loop pathways [ $\sim 55 \mathrm{msec}$ in the wrist flexor (Dick et al., 1987)]. Our results support the hypothesis of subthreshold activation of spinal circuitry during motor imagery. This is also consistent with previous reports of facilitatory effects during motor imagery using F-wave and H-reflex techniques (Bonnet et al., 1997; Kiers et al., 1997; Rossini et al., 1999).

\section{Potential mechanisms for enhanced spinal excitability}

There are several potential explanations for this imagery-induced modulation of stretch reflex at the spinal level. First, as indicated above, it could be because of subthreshold activation of motoneurons during motor imagery (cf. Gandevia et al., 1997). Because of the existence of direct corticomotoneuronal connections, especially for finger muscles (Porter and Lemon, 1993), it is possible that the excitability of spinal motoneurons is modulated directly by the motor cortex outflow.

Second, contributions may also arise from analogous modulation of spinal INs. These INs also receive direct inputs from M1 and higher premotor areas (Dum and Strick, 1991; He et al., 1995) as well as from brainstem nuclei under cortical control. Modulation of activities in INs was reported during an instructed delay period (an interval between a transient instruction cue and a subsequently triggered movement) in monkeys (Prut and Fetz, 1999). Specifically, approximately one-third of these INs showed significant delay modulation in the absence of EMG activities, a condition potentially analogous to that occurring during motor imagery tasks. Thus, an increase in the IN activities may occur during motor imagery. Last, a combination of the previous two mechanisms could potentially contribute to the observed effect.

Another possibility is that there is an enhanced excitability of 
the $\gamma$-motor system during motor imagery. Even without changes in the excitability of motoneurons and interneurons, the effect of an external stretch would thus be magnified and could trigger a stretch reflex during motor imagery. However, this is not likely to happen, because Gandevia et al. (1997) reported no change in spindle afferent discharge (and therefore the $\gamma$-motor system) during motor imagery. In contrast, their results showed increased motoneuronal activity and reflex excitability.

Besides the above-mentioned possible mechanisms of subthreshold activation, motoneurons could be actively inhibited from discharging through "parallel inhibitory mechanisms" (Takakusaki et al., 2001) despite increased corticospinal input during motor imagery.

\section{Comparison with $\mathrm{H}$-reflex and $\mathrm{F}$-wave responses}

Our results are also different from those obtained from H-reflex (Kasai et al., 1997; Hashimoto and Rothwell, 1999) and F-wave (Rossini et al., 1999; Facchini et al., 2002) techniques. The difference may lie in different techniques. The $\mathrm{F}$ wave is caused by antidromic activation of the motoneurons by strong electrical stimulation of peripheral nerves. Modulation of F waves may not be representative of the change of the entire $\alpha$ motoneuron pool (Mercuri et al., 1996). H-reflexes are evoked by electrical stimulation of peripheral group Ia afferents and recorded in the EMG from the muscle innervated by the stimulated nerve (Berardelli et al., 1987). They are thought to be mainly monosynaptic in origin and thus reflect the excitability of the spinal motoneurons as well as the transmission over the group Ia synapses on the motoneurons. Stretch reflexes originate primarily from spindle afferents (Ia and II) and also involve input from skin and subcutaneous nerves (Corden et al., 2000). Therefore, it appears that stretch reflexes use both monosynaptic and polysynaptic connections within the spinal cord, and some contributions may even be transcortical in origin (Goodin et al., 1990).

Together, it could be easier to detect changes in mechanically evoked stretch reflexes than electrically evoked $\mathrm{H}$ reflexes and $\mathrm{F}$ waves during motor imagery. Thus, the experimental paradigm used in this experiment has some advantages over previous approaches to test changes in spinal excitability during motor imagery.

\section{Differential effects on finger flexors and extensors}

The observation of different effects of motor imagery on the finger flexors and extensors is also of particular interest. Responses in the EDC were present in almost all cases (both ImFlex-FLEX and ImExt-FLEX) when this muscle was stretched. In contrast, responses in the FDS depended on the direction of the imagined movement: observed only in some subjects in the ImFlex-EXT tasks, not in the ImExt-EXT tasks at all. Differences in EMG responses were also accompanied by differences in torque responses. Furthermore, the onset latency of EMG responses was longer in EDC than in FDS. These differential effects suggest that imagined finger flexion and extension are not symmetrically controlled activities. This is similar to what has been observed during actual voluntary movements (Yue et al., 2000; Nakazawa et al., 2001).

\section{Conclusion}

Given general agreement regarding the involvement of primary motor cortex and the resulting enhanced corticospinal excitability in motor imagery tasks, our results of reflex response to stretches at the latency close to that induced by tendon taps provide evidence that motor imagery increases the excitability of spinal reflex pathways in a sustained subthreshold manner. We conclude that motor imagery exerts significant effects on spinal segmental circuitry.

\section{References}

Abbruzzese G, Berardelli A, Rothwell JC, Day BL, Marsden CD (1985) Cerebral potentials and electromyographic responses evoked by stretch of wrist muscles in man. Exp Brain Res 58:544-551.

Abbruzzese G, Trompetto C, Schieppati M (1996) The excitability of the human motor cortex increases during execution and mental imagination of sequential but not repetitive finger movements. Exp Brain Res 111:465-472.

Abbruzzese G, Assini A, Buccolieri A, Marchese R, Trompetto C (1999) Changes of intracortical inhibition during motor imagery in human subjects. Neurosci Lett 263:113-116.

Berardelli A, Day BL, Marsden CD, Rothwell JC (1987) Evidence favouring presynaptic inhibition between antagonist muscle afferents in the human forearm. J Physiol (Lond) 391:71-83.

Bonnet M, Decety J, Jeannerod M, Requin J (1997) Mental simulation of an action modulates the excitability of spinal reflex pathways in man. Brain Res Cogn Brain Res 5:221-228.

Capaday C, Forget R, Fraser R, Lamarre Y (1991) Evidence for a contribution of the motor cortex to the long-latency stretch reflex of the human thumb. J Physiol (Lond) 440:243-255.

Corden DM, Lippold OCJ, Buchanan K, Norrington C (2000) Long-latency component of the stretch reflex in human muscle is not mediated by intramuscular stretch receptors. J Neurophysiol 84:184-188.

Crago PE, Houk JC, Hasan Z (1976) Regulatory actions of human stretch reflex. J Neurophysiol 39:925-935.

Crammond DJ (1997) Motor imagery: never in your wildest dream. Trends Neurosci 20:54-57.

Decety J (1996a) The neurophysiological basis of motor imagery. Behav Brain Res 77:45-52.

Decety J, Jeannerod M, Prablanc C (1989) The timing of mentally represented actions. Behav Brain Res 34:35-42.

Dick JP, Rothwell JC, Day BL, Wise RJ, Benecke R, Marsden CD (1987) Modulation of the long-latency reflex to stretch by the supplementary motor area in humans. Neurosci Lett 75:349-354.

Dum RP, Strick PL (1991) The origin of corticospinal projections from the premotor area in the frontal lobe. J Neurosci 11:667-689.

Facchini S, Muellbacher W, Battaglia F, Boroojerdi B, Hallett M (2002) Focal enhancement of motor cortex excitability during motor imagery: a transcranial magnetic stimulation study. Acta Neurol Scand 105:146-151.

Fadiga L, Fogassi L, Pavesi G, Rizzolatti G (1995) Motor facilitation during action observation: a magnetic stimulation study. J Neurophysiol 73:2608-2611.

Fadiga L, Buccino G, Craighero L, Fogassi L, Gallese V, Pavesi G (1999) Corticospinal excitability is specifically modulated by motor imagery: a magnetic stimulation study. Neuropsychologia 37:147-158.

Gandevia SC, Wilson LR, Inglis JT, Burke D (1997) Mental rehearsal of motor tasks recruits alpha-motoneurones but fails to recruit human fusimotor neurones selectively. J Physiol (Lond) 505:259-266.

Goodin DS, Aminoff MJ, Shih PY (1990) Evidence that the long-latency stretch responses of the human wrist extensor muscle involve a transcerebral pathway. Brain 113:1075-7091.

Hashimoto R, Rothwell JC (1999) Dynamic changes in corticospinal excitability during motor imagery. Exp Brain Res 125:75-81.

He SQ, Dum RP, Strick PL (1995) Topographic organization of corticospinal projections from the frontal lobe: motor areas on the medial surface of the hemisphere. J Neurosci 15:3284-3306.

Jeannerod M (1995) Mental imagery in the motor context. Neuropsychologia 33:1419-1432.

Kamper DG, Rymer WZ (2000) Quantitative features of the stretch response of extrinsic finger muscles in hemiparetic stroke. Muscle Nerve 23:954-961.

Kamper DG, Schmit BS, Rymer WZ (2001) Effect of muscle biomechanics on the quantification of spasticity. Ann Biomed Eng 29:1122-1134.

Kasai T, Kawai S, Kawanishi M, Yahagi S (1997) Evidence for facilitation of motor evoked potentials (MEPs) induced by motor imagery. Brain Res 744:147-150.

Kiers L, Fernando B, Tomkins D (1997) Facilitatory effect of thinking about 
movement on magnetic motor-evoked potentials. Electroencephalogr Clin Neurophysiol 105:262-268.

Lewis GN, Polych MA, Byblow WD (2004) Proposed cortical and subcortical contributions to the long-latency stretch reflex in the forearm. Exp Brain Res 156:72-79.

Li S, Latash M, Zatsiorsky V (2004) Effects of motor imagery on finger force responses to transcranial magnetic stimulation. Brain Res Cog Brain Res $20: 273-280$

Lin FM, Sabbahi M (1998) The aging effects on the EMG and mechanical responses of the human wrist flexor stretch reflexes. Electromyogr Clin Neurophysiol 38:323-331.

Marsden CD, Merton PA, Morton HB (1976) Stretch reflex and servo action in a variety of human muscles. J Physiol (Lond) 259:531-560.

Mercuri B, Wassermann EM, Manganotti P, Ikoma K, Samii A, Hallett M (1996) Cortical modulation of spinal excitability: an F-wave study. Electroencephalogr Clin Neurophysiol 101:16-24.

Morita H, Olivier E, Baumgarten J, Petersen NT, Christensen LO, Nielsen JB (2000) Differential changes in corticospinal and Ia input to tibialis anterior and soleus motor neurones during voluntary contraction in man. Acta Physiol Scand 170:65-76.

Nakazawa K, Yamamoto SI, Ohtsuki T, Yano H, Fukunaga T (2001) Neural control: novel evaluation of stretch reflex sensitivity. Acta Physiol Scand $172: 257-268$

Petersen NT, Pyndt HS, Nielsen JB (2003) Investigating human motor control by transcranial magnetic stimulation. Exp Brain Res 152:1-16.
Porter R, Lemon RN (1993) Corticospinal function and voluntary movement. Oxford: Oxford UP.

Prut Y, Fetz EE (1999) Primate spinal interneurons show pre-movement instructed delay activity. Nature 401:590-594.

Rossini PM, Rossi S, Pasqualetti P, Tecchio F (1999) Corticospinal excitability modulation to hand muscles during movement imagery. Cereb Cortex 9:161-167.

Sirigu A, Cohen L, Duhamel JR, Pillon B, Dubois B, Agid Y, PierrotDeseilligny C (1995) Congruent unilateral impairments for real and imagined hand movements. NeuroReport 6:997-1001.

Takakusaki K, Kohyama J, Matsuyama K, Mori S (2001) Medullary reticulospinal tract mediating the generalized motor inhibition in cats: parallel inhibitory mechanisms acting on motoneurons and on interneuronal transmission in reflex pathways. Neuroscience 103:511-527.

Yahagi S, Kasai T (1999) Motor evoked potentials induced by motor imagery reveal a functional asymmetry of cortical motor control in left- and right-handed human subjects. Neurosci Lett 276:185-188.

Yue G, Cole KJ (1992) Strength increases from the motor program: comparison of training with maximal voluntary and imagined muscle contractions. J Neurophysiol 67:1114-1123.

Yue GH, Liu JZ, Siemionow V, Ranganathan V, Ng TC, Sahgal V (2000) Brain activation during human finger extension and flexion movements. Brain Res 856:291-300. 